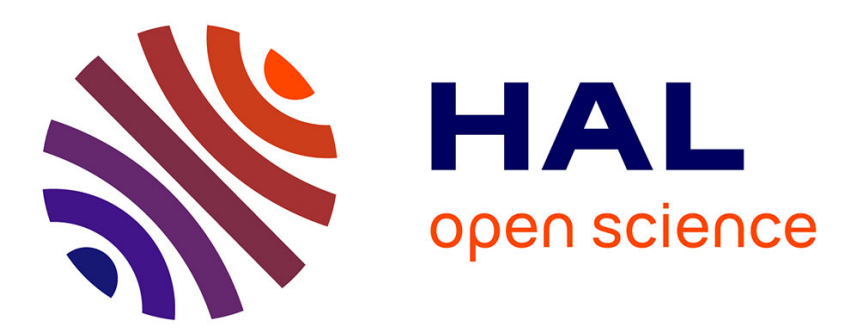

\title{
Formation Control of Agricultural Mobile Robots: A Bidirectional Weighted Constraints Approach
}

\author{
Audrey Guillet, Roland Lenain, Benoit Thuilot, Vincent Rousseau
}

\section{To cite this version:}

Audrey Guillet, Roland Lenain, Benoit Thuilot, Vincent Rousseau. Formation Control of Agricultural Mobile Robots: A Bidirectional Weighted Constraints Approach. Journal of Field Robotics, 2017, 34 (7), pp.1260 - 1274. 10.1002/rob.21704 . hal-01658323

\section{HAL Id: hal-01658323 \\ https://hal.uca.fr/hal-01658323}

Submitted on 16 May 2020

HAL is a multi-disciplinary open access archive for the deposit and dissemination of scientific research documents, whether they are published or not. The documents may come from teaching and research institutions in France or abroad, or from public or private research centers.
L'archive ouverte pluridisciplinaire HAL, est destinée au dépôt et à la diffusion de documents scientifiques de niveau recherche, publiés ou non, émanant des établissements d'enseignement et de recherche français ou étrangers, des laboratoires publics ou privés. 


\title{
Formation control of agricultural mobile robots: a bi-directional weighted constraints approach
}

\author{
Audrey Guillet \\ Irstea, \\ 9 avenue Blaise Pascal - CS 20085, \\ 63178 Aubière, France \\ audrey.guillet@irstea.fr \\ Benoit Thuilot \\ Institut Pascal, UMR 6602, \\ Campus des Cézeaux, \\ 24 avenue des Landais, BP 80026, \\ 63171 Aubière, France \\ benoit.thuilot@univ-bpclermont.fr
}

\author{
Roland Lenain \\ Irstea, \\ 9 avenue Blaise Pascal - CS 20085, \\ 63178 Aubière, France \\ roland.lenain@irstea.fr \\ Vincent Rousseau \\ Irstea, \\ 9 avenue Blaise Pascal - CS 20085, \\ 63178 Aubière, France \\ vincent.rousseau@irstea.fr
}

\begin{abstract}
This paper focuses on the formation control of several mobile robots in off-road conditions. A control strategy based on bidirectional referencing is proposed, where each robot combines a velocity control w.r.t. to the immediate preceding neighbor with a control w.r.t. to the immediate following neighbor. Two virtual leaders, respectively at the head and at the tail of the fleet, are propelling the fleet at the desired velocity. The fleet stability is investigated using Lyapunov techniques, pending on combination coefficients. The stability is theoretically proven for constant coefficients as weights of the respective velocity controls and tested through simulations and full-scale experiments. It is shown that this control strategy permits to reduce the error propagation problem often encountered in formation control, while limiting the communication problems of the global strategies. As a result, a stable formation control architecture is defined, which requires each vehicle to be refereed only to two other robots. This limited communication need is particularly interesting for agricultural operations. The proposed approach is implemented in this paper on agricultural tractors.
\end{abstract}

\section{Introduction}

Cooperation of several vehicles presents a lot of applications whether for exploration, mapping, or also for area coverage in surveillance. It is particularly used in agriculture in order to achieve several operations during the same passage or extend the work of a single machine. These operations performed by a cooperation of several autonomous mobile robots may be considered in order to limit the workforce needed as well as the arduousness. As pointed out for instance in (Blackmore, 2014), such an approach may permit to open the way to new production tools, composed of a collection of small autonomous mobile robots, acting together in order to achieve significant agriculture work. In order to be applicable, the coordinated motion of the robots has to be stable and precise during the travel along a common path. In a decentralized framework, this is ensured by the way each robot computes its control laws. The main control strategies for multi-robot 
path tracking in formation can be classified in three main types: behavior-based, virtual structure control and leader-follower. The behavior-based control defines different objectives for the robots (typically goal attraction, formation keeping and neighbors avoidance), which are weighted to obtain the local command for each robot (see for instance (Balch and Arkin, 1998), (Baras et al., 2003)). However, at the global level, the behavior of the whole fleet is difficult to predict. Therefore, the coordinated motion required for given applications, such as in agriculture, cannot be achieved. In the virtual control structure strategy, the set of robots defines a structure which state is controlled (usually through its center of mass defining a virtual leader) and then transformed as a local control for each robot (see (Antonelli et al., 2013), (Ren and Beard, 2004)). This strategy is of interest in centralized control when a controller knows the position of all robots. However, decentralized control using this strategy requires important communication network as each robot has to know the state of all other robots of the fleet. This may be penalizing for agricultural applications, since distance between robots may be important and communication delays are consequently not negligible. The strategy limiting the number of communications is the leader-follower approach (see for instance (Kowdiki et al., 2012), or (Qian et al., 2015)). In this point of view, each robot regulates its position and orientation with respect to another robot named leader. The choice of the reference robot for each robot influences the global behavior of the overall formation. A classical control strategy consists in referencing all the robots to a common one, called the global leader of the fleet, which then carries the desired velocity for the tracking. This strategy generates a very stable behavior with limited communication links $(n-1$ links for $n$ robots in the formation), but it is also very dependent on a unique robot, the leader, and it cannot guarantee the safety of the follower robots, as they have no information about the position of their surrounding fellows.

To overcome these safety issues, another strategy considered is for each robot to use a different local leader, typically to take as reference its direct preceding fellow, as achieved for highway platooning in (Khatir and Davison, 2004) or in (Klančar et al., 2011). Given that each robot is regulated to the closest and most dangerous one in case of failure, the robots safety is ensured at each step. However, the global behavior of the whole fleet exhibits oscillations between head and tail due to error accumulation in the successive positioning regulations. The addition of a predictive overlay as proposed in (Farrokhsiar and Najjaran, 2012) or (Guillet et al., 2014) permits to anticipate for some of those errors and reduce the servoing overshoots. Even so, for fleet larger than 3 robots, the oscillation effect is still observable and prevent the direct application of this method. Therefore, to further improve the stability of the servoing, we propose here to use a double referencing to both the previous and the following robot, with two leaders at the head and tail of the fleet. This reduces the dependency to a unique vulnerable leader and permits to have a symmetrical behavior of the formation, from the head to the tail but also from the tail to the head, while keeping limited and local communication links. In (Lafferriere et al., 2005), (Yamaguchi et al., 2001), or in (Zhang et al., 1999), this bi-directional control strategy is considered in the particular case of reciprocal interactions between agents. In this paper, we expand it to more general asymmetrical relations cases, studying the global stability and observing the effects on the whole behavior of the fleet.

The formation is defined and the control laws are presented in the second part of this paper. The third part focuses on proving the stability of the formation using Lyapunov method, firstly for any configuration of a 3-vehicle formation, then in a general n-vehicle formation for two particular cases of relative weights. Simulations for a fleet of 5 vehicles are presented in part four. Differents full-scale experiments were also conducted using 3 wheeled mobile robots and results are presented in the fifth part.

\section{Modeling and control of the robot formation}

In this paper, we consider a fleet of mobile robots composed of $n$ mobile robots, tracking a known path named $\Gamma$. It is wished that the fleet maintains a potentially varying formation defined as a set of desired distances between robots. In the local frame of the path, the desired formation shape is expressed as a desired lateral distance to the path $y_{i}^{d e s}$ for each robot $i$ and a desired longitudinal curvilinear distance (i.e. along the reference trajectory) $d_{i / j}^{\text {des }}$ between robots $i$ and $j$, as illustrated in Figure 1. 


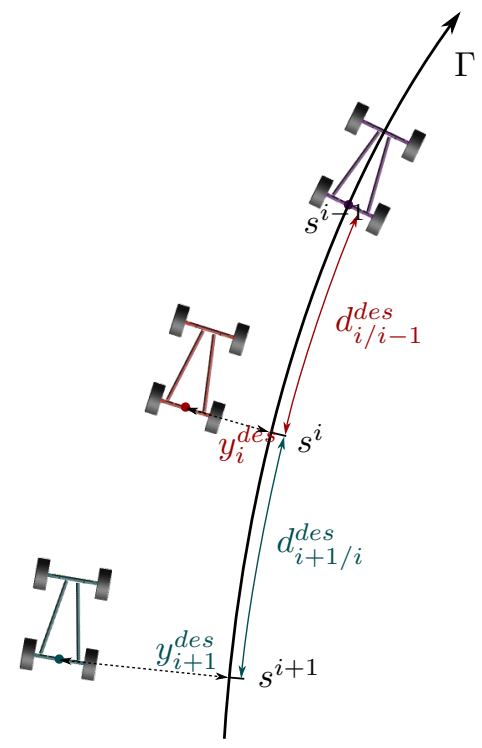

Figure 1: Formation shape definition

Without loss of generality, car-like mobile robots are considered here (known as 1,1 in classification proposed in (Campion et al., 1996)). For the modeling, each vehicle-like robot is simplified as a bicycle controlled by its front steering angle $\delta$ and its velocity $v$ (each robot is supposed to be symmetrical). It is fitted with sensors providing an absolute positioning, which is then transformed into a local positioning with respect to the reference trajectory $\Gamma$. In the local frame, the robot's state is characterized by:

- its lateral deviation $y$, distance to the closest point $M$ of the path,

- its curvilinear coordinate $s$ of $M$, defined as the distance traveled along the path,

- its angular error $\tilde{\theta}$, difference between the tangent to the trajectory at the point $M$ and the robot heading.

Additionally, the path $\Gamma$ being known, the curvature $c(s)$ of the path at the point $\mathrm{M}$ can also be deduced. In order to model the actual motion of the robot, two additional angles $\beta^{F}$ and $\beta^{R}$ are introduced as the difference between the velocity direction and the tire direction of each wheel. These angles, named sideslip angles, reflect the skidding of the wheel on the ground, which is a significant phenomenon when the robot runs on an uneven terrain in off-road conditions (Wang and Low, 2007). As these angles are hardly measurable, their value is estimated on-line by means of an observer which design is detailed in (Lenain et al., 2014).

With these notations, illustrated in Figure 2, the kinematic evolution of the robot is expressed as:

$$
\left\{\begin{array}{l}
\dot{s}=v \frac{\cos \left(\tilde{\theta}+\beta^{R}\right)}{1-c(s) y} \\
\dot{y}=v \sin \left(\tilde{\theta}+\beta^{R}\right) \\
\dot{\tilde{\theta}}=v\left(\cos \left(\beta^{R}\right) \frac{\tan \left(\delta+\beta^{F}\right)-\tan \left(\beta^{R}\right)}{L}-\frac{c(s) \cos \left(\tilde{\theta}+\beta^{R}\right)}{1-c(s) y}\right)
\end{array}\right.
$$

It should be noted that this model is defined as long as $1-c(s) . y \neq 0$, which means that the lateral position of the robot should not be superimposed with the instantaneous center of rotation $A$ of the path. This assumption is experimentally guaranteed given the small curvatures of the path compared with the desired lateral deviations.

The model (1), in which all variables are known either by measure or by estimation, can then be used to 


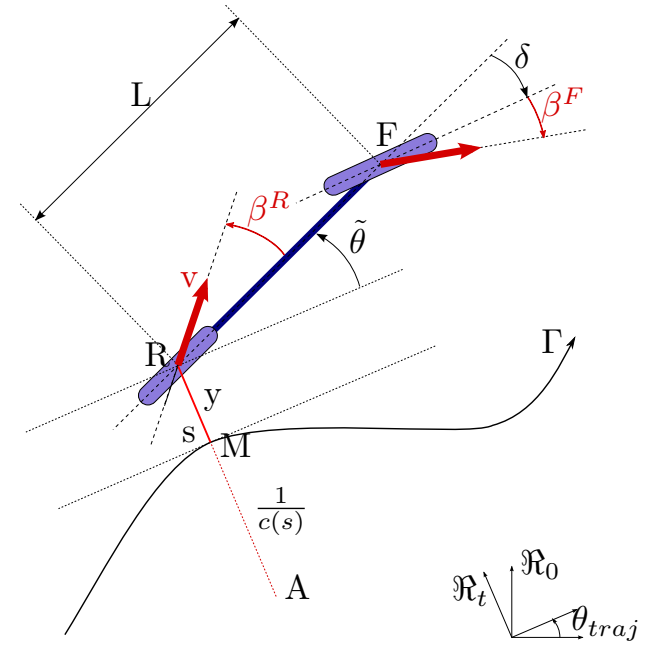

Figure 2: Model of a robot in the local frame

design the control laws for the regulation of the lateral and longitudinal position of the robot with respect to the reference path and the other robots of the formation.

\subsection{Lateral servoing}

The lateral servoing aims to regulate the lateral distance $y_{i}$ of the $i t h$ robot to the reference path to the desired distance $y_{i}^{\text {des }}$ (possibly variable) through the control of the front steering angle $\delta_{i}$. From the extended kinematic model (1) of the robot, an invertible change of variables permits to transform it into a chained system (as detailed in (Samson, 1995)). In particular the state and control variables transformation:

$$
\begin{aligned}
{\left[s_{i}, y_{i}, \tilde{\theta}_{i}\right] } & \rightarrow\left[x_{1}^{i}, x_{2}^{i}, x_{3}^{i}\right]=\left[s_{i}, y_{i},\left(1-c\left(s_{i}\right) y_{i}\right) \tan \left(\tilde{\theta}_{i}+\beta_{i}^{R}\right)\right] \\
{\left[v_{i}, \delta_{i}\right] } & \rightarrow\left[m_{1}^{i}, m_{2}^{i}\right]=\left[\frac{v_{i} \cos \left(\tilde{\theta}_{i}+\beta_{i}^{R}\right)}{1-c\left(s_{i}\right) y_{i}}, \frac{d x_{3}^{i}}{d t}\right]
\end{aligned}
$$

leads the extended kinematic model (1) of robot $i$ to become model (3):

$$
\left\{\begin{aligned}
\dot{x}_{1}^{i} & =m_{1}^{i} \\
\dot{x}_{2}^{i} & =x_{3}^{i} \\
\dot{x}_{3}^{i} & =\frac{d x_{3}^{i}}{d t}=m_{2}^{i}
\end{aligned}\right.
$$

This model is almost linear and rely on the time derivative of the state of the robot. In order to achieve the control of a formation for agricultural operations, it is interesting to derive independently lateral and longitudinal dynamics of each robot. This indeed allows to consider a decoupled relative servoing of each robot with respect to other robots along the path to be followed (i.e. using curvilinear distances and desired lateral deviations). In order to do so, let us consider the state derivative with respect to curvilinear abscissa instead of time derivative. This turns system (3) into the linear system (4).

$$
\left\{\begin{aligned}
x_{1}^{\prime i} & =1 \\
x_{2}^{\prime i} & =x_{3}^{i} \\
x_{3}^{\prime i} & =m_{3}^{i}=\frac{m_{2}^{i}}{m_{1}^{i}}
\end{aligned}\right.
$$

As detailed in (Lenain et al., 2006), the objective of the lateral control is to derive a virtual control $m_{3}^{i}$ for 
the $i t h$ robot in order to regulate its lateral deviation $y_{i}=x_{2}^{i}$ to the desired distance $y_{i}^{\text {des }}$. This can be obtained by imposing

$$
m_{3}^{i}=-K_{d} \varepsilon_{i}^{\prime y}-K_{p} \varepsilon_{i}^{y}+y_{i}^{\prime \prime d e s} \quad\left(K_{d}, K_{p}>0\right)
$$

with $\varepsilon_{i}^{y}=y_{i}-y_{i}^{\text {des }}$. Such a virtual control indeed leads to the following differential equation of the lateral error with respect to $x_{1}^{i}=s^{i}$ :

$$
\varepsilon_{i}^{\prime \prime y}+K_{d} \varepsilon_{i}^{\prime y}+K_{p} \varepsilon_{i}^{y}=0
$$

From the expression of virtual control (5), and using the inverse of the transformation (2), the control law for the steering angle $\delta_{i}$ of the robot $i$ can be computed, leading to (7).

$$
\delta_{i}=\arctan \left[\tan \beta_{i}^{R}+\frac{L_{i}}{\cos \beta_{i}^{R}}\left(\frac{c\left(s_{i}\right) \cos \gamma_{i}}{1-c\left(s_{i}\right) y_{i}}+\frac{A_{i} \cos ^{3} \gamma_{i}}{\left(1-c\left(s_{i}\right) y_{i}\right)^{2}}\right)\right]-\beta_{i}^{F}
$$

where:

- $\gamma_{i}=\tilde{\theta}_{i}+\beta_{i}^{R}$

- $A_{i}=K_{p} \varepsilon_{i}+\left(1-c\left(s_{i}\right) y_{i}\right)\left[K_{d}\left(\tan \gamma_{i}-\frac{\dot{y}_{i}^{\text {des }}}{v_{i} \cos \gamma_{i}}\right)+c\left(s_{i}\right) \tan ^{2} \gamma_{i}\right]$

- $\left(K_{p}, K_{d}\right)$ are negative scalars designing the expected lateral dynamics

This control law, acting on the front steering angle allows to regulate the lateral deviation of the robot $i$ to the desired distance $y_{i}^{\text {des }}$, whatever the grip conditions (accounted for with the observation of the sideslip angles $\beta_{i}^{F}$ and $\beta_{i}^{R}$ ), and whatever the variations of $y_{i}^{d e s}$. This latter point is particularly interesting for agricultural operations, since the robot may be deployed in "wing" configuration (for extending agricultural work) and be in a singe file when achieving a half turn at the end of a row. Moreover, the desired lateral deviation may be adapted on-line according to other robots lateral error in order to maintain a desired shape for the fleet.

As achieved in (Lenain et al., 2006) for a single robot, the control law (7) may be enhanced with a predictive layer on the curvature $c\left(s_{i}\right)$ in order to tackle the actuator settling time and possible inertial effects. Such anticipation is not detailed in this paper. Finally it is important to note that the lateral control law is based on a robot evolution defined using derivative with respect to curvilinear abscissa $\left(s_{i}\right)$ instead of time derivative. Consequently, the tuning gains $\left(K_{p}, K_{d}\right)$ define a settling distance instead of a settling time. As a result, the lateral behavior of the robot is independent from its velocity. The longitudinal control of each robot may thus be computed separately.

\subsection{Longitudinal target tracking}

Given the above results, the longitudinal control law can be considered independently, assuming the lateral regulation is guaranteed by the lateral control law. An elementary control law is first designed. It derives a control velocity $v_{t}^{i}$ for the robot $i$ regulating its curvilinear distance to a target robot $t$ to the desired distance $d_{i / t}^{d e s}$. It is assumed that the position and state of the target is known by the controlled robot, communicated by the former. From their respective position, the interdistance error is defined

$$
\varepsilon_{t}^{i}=s^{t}-s^{i}-d_{i / t}^{d e s}
$$

Through the differentiation of the error equation (8), an exponential convergence of the longitudinal positioning error

$$
\dot{\varepsilon}_{t}^{i}=-k_{v} \cdot \varepsilon_{t}^{i}, \quad \text { with } \quad k_{v} \in \mathbb{R}^{+*}
$$

can be obtained by the definition of the velocity control law

$$
v_{t}^{i}=\frac{1-c\left(s^{i}\right) y_{i}}{\cos \left(\tilde{\theta}_{i}+\beta_{i}^{R}\right)}\left(v^{t} \frac{\cos \left(\tilde{\theta}_{t}+\beta_{t}^{R}\right)}{1-c\left(s^{t}\right) y_{t}}+k_{v} \cdot \varepsilon_{t}^{i}\right)
$$


This velocity control can also be expressed as a velocity along the path $\dot{s}_{t}^{i}$, called curvilinear velocity control

$$
\dot{s}_{t}^{i}=\dot{s}^{t}+k_{v} \cdot \varepsilon_{t}^{i}
$$

In order to compensate for the response behavior of the real robots low-level, which creates a disparity between the velocity command and the actual response, a predictive algorithm is added. In a first step, given the settling time of the actuators, the future positions of the robot and the target are estimated (from their current state). Next, the velocity control law (10) applied to the future positions provides a velocity objective after the prediction horizon. Eventually, the behavior of the actuators is accounted for through a minimization algorithm which derives the optimal control velocity so that the response of the system reaches the desired velocity objective. This control law has been experimentally tested using wheeled mobile robots in off-road conditions in (Guillet et al., 2013) and results exhibit that the dynamics are well accounted for as they do not alter the servoing and the exponential convergence is practically shown.

\subsection{Bi-directional formation control strategy}

The formation control strategy for the fleet consists then in defining the links between the robots of the formation, and thus the target(s) of each robot for the computation of its velocity command. In the formation framework proposed, each robot interacts with its two closest neighbors, the immediate preceding robot and the immediate following one. The formation graph, illustrated in Figure 3, presents the bi-directional links between neighbors robots. However, they are not assimilated to unidirectional links as the influences of the neighboring robots $i$ and $j$ on each other are independent and asymmetrical, characterized by the coefficients $\mu_{j}^{i}$ and $\mu_{i}^{j}$ (with $\mu_{j}^{i}$ independent of $\mu_{i}^{j}$ ). The first and last robots of the formation do not have an immediate
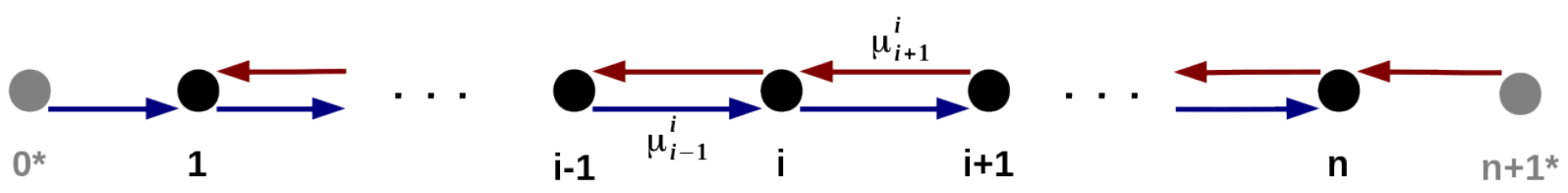

Figure 3: Formation graph with virtual leaders $(*)$ and control coefficients

preceding fellow (respectively an immediate following one). Hence, we define two virtual leaders (illustrated with a * in Figure 3) with which the extreme robots interact, and which carry the desired global velocity for the whole fleet.

A robot $i$ uses the control law presented in Subsection 2.2 to compute a command $\dot{s}_{i-1}^{i}$ ensuring the servoing with respect to the preceding robot $i-1$, and another command $\dot{s}_{i+1}^{i}$ ensuring the servoing with respect to the following robot $i+1$. These two velocities are linearly combined to get the final velocity command $\dot{s}^{i}$ for the $i t h$ robot:

$$
\dot{s}^{i}=\mu_{i-1}^{i} \cdot \dot{s}_{i-1}^{i}+\mu_{i+1}^{i} \cdot \dot{s}_{i+1}^{i}
$$

The control coefficients in the final control law (12) represent the relative weight of each elementary control and must therefore verify $\left(\mu_{i-1}^{i}, \mu_{i+1}^{i}\right) \in[0,1]^{2}$ with $\mu_{i-1}^{i}+\mu_{i+1}^{i}=1$.

As for the first robot (respectively the last one), it combines a classical velocity control law compensating the interdistance error w.r.t. to its following (respectively preceding) neighbor with the desired traveling velocity $\dot{s}^{C}$, i.e. $\dot{s}_{0}^{1}=\dot{s}_{n+1}^{n}=\dot{s}^{C}$.

\section{Stability of a n-vehicle formation}

Let us consider a fleet of $n$ vehicles controlled by the bidirectional control strategy previously defined. The formation is asymptotically stable iff the positioning errors of each vehicle $\varepsilon_{j}^{i}$ converge to zero. To characterize 
the evolution of the formation, let us express the evolution of each robot. From (11) and (12), one can write

$$
\dot{s}^{i}=\mu_{i-1}^{i} \cdot\left(\dot{s}^{i-1}+k \cdot \varepsilon_{i-1}^{i}\right)+\mu_{i+1}^{i} \cdot\left(\dot{s}^{i+1}+k \cdot \varepsilon_{i+1}^{i}\right)
$$

or

$$
\dot{s}^{i}-\mu_{i-1}^{i} \cdot \dot{s}^{i-1}-\mu_{i+1}^{i} \cdot \dot{s}^{i+1}=k \cdot\left(\mu_{i-1}^{i} \cdot \varepsilon_{i-1}^{i}-\mu_{i+1}^{i} \cdot \varepsilon_{i}^{i+1}\right)
$$

By definition of the formation control strategy for the first (respectively the last) robot of the fleet, the partial curvilinear velocity control wrt. the previous robot (respectively velocity to the following robot) is defined as the desired travel velocity along the path $\dot{s}^{C}$. The evolution of the whole fleet can be expressed from (14), with the vectors

$$
\begin{aligned}
& \dot{\boldsymbol{s}}=\left[\dot{s}^{1}, \ldots, \dot{s}^{n}\right]^{T} \\
& \varepsilon=\left[\varepsilon_{1}^{2}, \ldots, \varepsilon_{n-1}^{n}\right]^{T}
\end{aligned}
$$

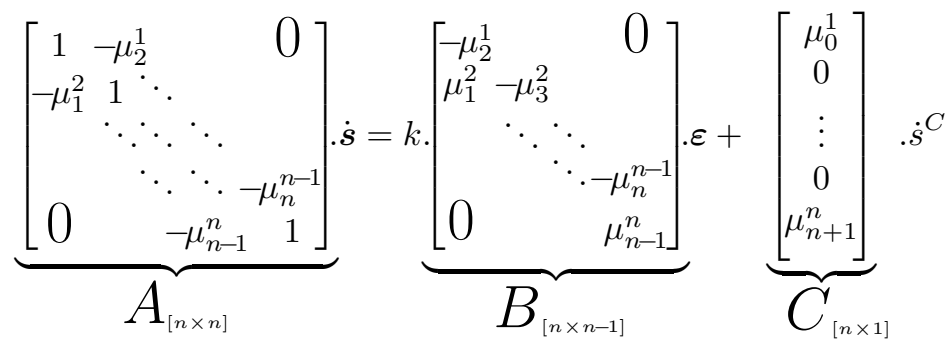

A is a non-symmetrical tridiagonal matrix with diagonal elements equal to 1 . From the properties of such tridiagonal matrices (see (El-Mikkawy and Karawia, 2006)), the determinant satisfies the recurrence relationship

$$
\begin{aligned}
\operatorname{det}(A) & =\operatorname{det}\left(A_{n-1}\right)-\mu_{n-1}^{n} \cdot \mu_{n}^{n-1} \cdot \operatorname{det}\left(A_{n-2}\right) \\
& =\operatorname{det}\left(A_{n-1}\right)-\left(1-\mu_{n+1}^{n}\right) \cdot \mu_{n}^{n-1} \cdot \operatorname{det}\left(A_{n-2}\right)
\end{aligned}
$$

with $A_{n-i}$ the sub-matrix of the $n-i$ first columns and rows of $A$.

It is later verified that the choice of the coefficients $\mu_{j}^{i}$ leads the matrix $A$ to be non-singular, and therefore invertible.

In general case for coefficients $\mu_{j}^{i}$, the coefficients $a_{i j}^{-1}$ of the matrix $A^{-1}$, inverse of $A$, are:

$$
\left\{\begin{aligned}
a_{11}^{-1} & =\left(1-\mu_{1}^{2} \mu_{2}^{1} \frac{\beta_{3}}{\beta_{2}}\right)^{-1}=\left(\frac{\beta_{1}}{\beta_{2}}\right)^{-1}=\frac{\beta_{2}}{\beta_{1}} \\
a_{n n}^{-1} & =\left(1-\mu_{n-1}^{n} \mu_{n}^{n-1} \frac{\alpha_{n-2}}{\alpha_{n-1}}\right)^{-1}=\left(\frac{\alpha_{n}}{\alpha_{n-1}}\right)^{-1}=\frac{\alpha_{n-1}}{\alpha_{n}} \\
a_{i i}^{-1} & =\left(1-\mu_{i-1}^{i} \mu_{i}^{i-1} \frac{\alpha_{i-2}}{\alpha_{i-1}}-\mu_{i}^{i+1} \mu_{i+1}^{i} \frac{\beta_{i+2}}{\beta_{i+1}}\right)^{-1} \quad \text { for } i=\{2,3, \ldots, n-1\} \\
a_{i j}^{-1} & =\left(\prod_{k=i}^{j-1} \mu_{k+1}^{k}\right) \frac{\alpha_{i-1}}{\alpha_{j-1}} a_{j j}^{-1} \\
i<j & \left(\prod_{k=j+1}^{i} \mu_{k-1}^{k}\right) \frac{\beta_{i+1}}{\beta_{j+1}} a_{j j}^{-1}
\end{aligned}\right.
$$

with the two vectors $\boldsymbol{\alpha}=\left(\alpha_{0}, \alpha_{1}, \ldots, \alpha_{n}\right)$ and $\boldsymbol{\beta}=\left(\beta_{1}, \beta_{2}, \ldots, \beta_{n+2}\right)$ defined by recurrence relationships as:

$$
\begin{aligned}
& \alpha_{i}= \begin{cases}1 & \text { if } i=0,1 \\
\alpha_{i-1}-\mu_{i-1}^{i} \mu_{i}^{i-1} \alpha_{i-2} & \text { if } i \geq 2\end{cases} \\
& \beta_{i}= \begin{cases}1 & \text { if } i=n+1, n \\
\beta_{i+1}-\mu_{i+1}^{i} \mu_{i}^{i+1} \beta_{i+2} & \text { if } i \leq n-1\end{cases}
\end{aligned}
$$


Given the expression of the matrices, the evolution of the position of the robots of the formation can be derived from (15)

$$
\dot{s}=k \cdot A^{-1} \cdot B \cdot \varepsilon+A^{-1} \cdot C \cdot \dot{s}^{C}
$$

In order to study the formation stability, let us consider the positive-definite Lyapunov candidate:

$$
V(\varepsilon)=\frac{1}{2} \varepsilon^{T} \cdot \varepsilon
$$

The differentiation gives

$$
\dot{V}(\varepsilon)=\varepsilon^{T} . \dot{\varepsilon}
$$

From (8), one can derive the evolution of the longitudinal errors

$$
\dot{\varepsilon}=\underbrace{\left[\begin{array}{cccccc}
1 & -1 & & & 0 & \\
& 1 & -1 & & & \\
& & \ddots & \ddots & & \\
& & & 1 & -1 & \\
& & & & 1 & -1
\end{array}\right]}_{D_{[n-1 \times n]}} . \dot{s}
$$

Hence, from (21), (24) can be rewritten as

$$
\dot{\varepsilon}=k \cdot D \cdot A^{-1} \cdot B \cdot \varepsilon+D \cdot A^{-1} \cdot C \cdot \dot{s}^{C}
$$

and the Lyapunov derivative function (23) is therefore:

$$
\dot{V}(\varepsilon)=k \cdot \varepsilon^{T} \cdot \underbrace{D \cdot A^{-1} \cdot B}_{\mathrm{M}} \cdot \varepsilon+\varepsilon^{T} \cdot \underbrace{D \cdot A^{-1} \cdot C}_{\mathrm{N}} \cdot \dot{s}^{C}
$$

The stability property of the formation is driven by the properties of the matrices $M$ and $N$, which depend on the combination coefficients $\mu_{j}^{i}$ of the bidirectional control law. Let us examine the properties of the Lyapunov derivative function in particular cases.

\subsection{Variable coefficients in a 3-vehicle formation}

In a first case, let us consider a reduced formation of 3 vehicles. The first and third vehicles are linked to the virtual leaders, while the second vehicle is referencing to the two extremes ones. The set of combination coefficients $\left(\mu_{i-1}^{i}\right.$ and $\left.\mu_{i+1}^{i}\right)$ are independent between the 3 robots $i$ and they are not assumed to take any predefined or particular values.

With this formation definition, Equation (15) becomes

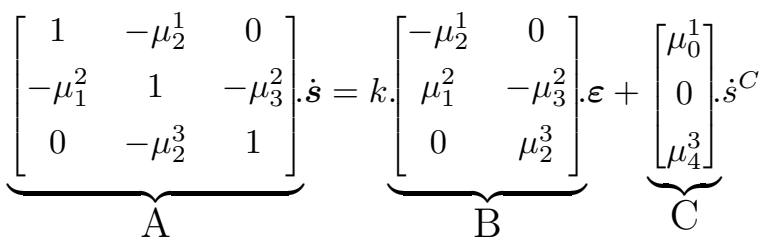

with the determinant of matrix $A$

$$
\begin{aligned}
\operatorname{det}(A) & =1-\mu_{1}^{2} \cdot \mu_{2}^{1}-\mu_{2}^{3} \cdot \mu_{3}^{2} \\
& =\mu_{0}^{1} \cdot \mu_{1}^{2}+\mu_{3}^{2} \cdot \mu_{4}^{3} \quad \geq 0
\end{aligned}
$$


Therefore, the matrix $A$ is non-singular as long as the coefficients, constants or variables, are non-null.

$A$ being invertible, the Lyapunov derivative function (26) can be expressed, with its matrices $M$ and $N$

$$
\dot{V}(\varepsilon)=k \cdot \varepsilon^{T} \cdot M \cdot \varepsilon+\varepsilon^{T} \cdot N \cdot \dot{s}^{C}
$$

where

$$
N=D \cdot A^{-1} \cdot C \quad \text { and } \quad M=D \cdot A^{-1} \cdot B
$$

The calculation of these matrices gives

$$
\begin{aligned}
N & =\left[\begin{array}{ccc}
1 & -1 & 0 \\
0 & 1 & -1
\end{array}\right] \cdot\left[\begin{array}{ccc}
1 & -\mu_{2}^{1} & 0 \\
-\mu_{1}^{2} & 1 & -\mu_{3}^{2} \\
0 & -\mu_{2}^{3} & 1
\end{array}\right]^{-1} \cdot\left[\begin{array}{c}
\mu_{0}^{1} \\
0 \\
\mu_{4}^{3}
\end{array}\right] \\
& =\mathbf{0}_{2 \times 1}
\end{aligned}
$$

and

$$
\begin{aligned}
M & =\left[\begin{array}{ccc}
1 & -1 & 0 \\
0 & 1 & -1
\end{array}\right] \cdot\left[\begin{array}{ccc}
1 & -\mu_{2}^{1} & 0 \\
-\mu_{1}^{2} & 1 & -\mu_{3}^{2} \\
0 & -\mu_{2}^{3} & 1
\end{array}\right]^{-1} \cdot\left[\begin{array}{cc}
-\mu_{2}^{1} & 0 \\
\mu_{1}^{2} & -\mu_{3}^{2} \\
0 & \mu_{2}^{3}
\end{array}\right] \\
& =\frac{1}{\operatorname{det}(A)}\left[\begin{array}{cc}
-\mu_{0}^{1} \mu_{1}^{2}-\mu_{2}^{1} \mu_{3}^{2} \mu_{4}^{3} & \mu_{0}^{1} \mu_{3}^{2} \mu_{4}^{3} \\
\mu_{0}^{1} \mu_{1}^{2} \mu_{4}^{3} & -\mu_{3}^{2} \mu_{4}^{3}-\mu_{0}^{1} \mu_{1}^{2} \mu_{2}^{3}
\end{array}\right]
\end{aligned}
$$

The eigenvalues of $M$ are

$$
\left\{\lambda_{1}, \lambda_{2}\right\}=\left\{-1,-\frac{\mu_{0}^{1} \mu_{1}^{2} \mu_{2}^{3}+\mu_{2}^{1} \mu_{3}^{2} \mu_{4}^{3}}{\operatorname{det}(A)}\right\}
$$

The final expression of (30) is simplified as

$$
\dot{V}(\varepsilon)=k \cdot \varepsilon^{T} \cdot M \cdot \varepsilon
$$

with $\mathrm{M}$ a negative semi-definite matrix.

If the combination coefficients are chosen so that

$$
\forall(i \in 1,2,3, j \in\{i-1, i+1\}), \quad \mu_{j}^{i} \neq 0 \Longrightarrow\left\{\begin{array}{rl}
\operatorname{det}(A) & \neq 0 \\
\mu_{2}^{1} \mu_{3}^{2} \mu_{4}^{3} & \neq 0 \\
\mu_{0}^{1} \mu_{1}^{2} \mu_{2}^{3} & \neq 0
\end{array} \Longrightarrow \lambda_{2}<0\right.
$$

Both eigenvalues of $\mathrm{M}$ are strictly negative, i.e. $\mathrm{M}$ is Hurwitz. Hence, the system, here the formation of 3 vehicles, is asymptotically stable regardless of the precise values of the coefficients.

\subsection{Symmetrical and reciprocal relations}

For a more general $n$-vehicle formation, the stability proof is detailed for two cases of constraint coefficients, firstly for symmetrical relations and then for constant asymmetrical relations.

In case of constant symmetrical relations, i.e.

$$
\forall i \in\{1,2, \ldots, n\}, \quad \mu_{\{i-1, i+1\}}^{i}=\frac{1}{2}
$$


the determinant of $A$ satisfies the relationship

$$
\operatorname{det}(A)=\frac{n+1}{2^{n}}
$$

Proof. $\quad$ for $n=1, \operatorname{det}\left(A_{1}\right)=1=\frac{n+1}{2^{n}}$

- for $n=2, \operatorname{det}\left(A_{2}\right)=1-\frac{1}{2} \frac{1}{2}=\frac{3}{4}=\frac{n+1}{2^{n}}$

- for $n>2$, if $\operatorname{det}\left(A_{n-2}\right)$ and $\operatorname{det}\left(A_{n-1}\right)$ verify the equation (37), then from (16) there is

$$
\begin{aligned}
\operatorname{det}\left(A_{n}\right) & =\operatorname{det}\left(A_{n-1}\right)-\mu_{n-1}^{i} \cdot \mu_{n}^{n-1} \cdot \operatorname{det}\left(A_{n-2}\right)=\frac{n}{2^{n-1}}-\frac{1}{2} \cdot \frac{1}{2} \cdot \frac{n-1}{2^{n-2}}=\frac{n}{2^{n-1}}-\frac{n-1}{2^{n}} \\
\operatorname{det}\left(A_{n}\right) & =\frac{n+1}{2^{n}}
\end{aligned}
$$

As a result, $\operatorname{det}\left(A_{n}\right)$ also verifies the relationship (37).

The matrix $A$ is really always non-singular; it can be inversed and the elements of $A^{-1}$ are

$$
\left\{\begin{array}{c}
a_{i j}^{-1}=\frac{2 i(n-j+1)}{n+1} \\
a_{i \leqslant j}^{-1}=\frac{2 j(n-i+1)}{n+1}
\end{array}\right.
$$

The matrices $M$ and $N$ in the derivative of the Lyapunov function (26) can be derived, with

$$
N=D \cdot A^{-1} \cdot C
$$

$A^{-1} . C$ is a vector of dimension $n$ and the $i t h$ element is

$$
\begin{aligned}
\left(A^{-1} \cdot C\right)_{i} & =\mu_{0}^{1} \cdot a_{i 1}^{-1}+\mu_{n+1}^{n} \cdot a_{i n}^{-1} \\
& =\frac{1}{2}\left(\frac{2(n-i+1)}{n+1}+\frac{2 i}{n+1}\right) \\
& =1
\end{aligned}
$$

$A^{-1} . C$ is the unity vector and therefore, given the structure of the matrix $D$, it can be easily deduced that

$$
N=D \cdot A^{-1} \cdot C=\mathbf{0}_{(n-1) \times 1}
$$

The Lyapunov derivative function is thus reduced to

$$
\dot{V}(\varepsilon)=k \cdot \varepsilon^{T} \cdot M \cdot \varepsilon
$$

As for the matrix $M$, its dimension is $(n-1, n-1)$, and it is expressed as

$$
\begin{aligned}
M & =D \cdot A^{-1} \cdot B \\
& =\left[\begin{array}{cccc}
1 & -1 & & \\
\ddots & \ddots & & \\
& \ddots & \ddots & \\
& & 1 & -1
\end{array}\right]\left[\begin{array}{cccc}
a_{11}^{-1} & \ldots & \ldots & a_{1 n}^{-1} \\
\vdots & \ddots & & \vdots \\
\vdots & & \ddots & \vdots \\
a_{n 1}^{-1} & \ldots & \ldots & a_{n n}^{-1}
\end{array}\right]\left[\begin{array}{ccc}
-\frac{1}{2} & \\
\frac{1}{2} & \ddots & \\
& \ddots & -\frac{1}{2} \\
& & \frac{1}{2}
\end{array}\right] \\
& =-\frac{1}{2} D \cdot A^{-1} \cdot D^{T}
\end{aligned}
$$


The detail of this expression gives, for $(i, j) \in\{1,2, \ldots, n-1\}^{2}$

$$
\begin{gathered}
m_{i j}=-\frac{1}{2}\left[\left(a_{i j}^{-1}-a_{i(j+1)}^{-1}\right)-\left(a_{(i+1) j}^{-1}-a_{(i+1)(j+1)}^{-1}\right)\right]= \begin{cases}\frac{1}{n+1} & \text { if } i<j \\
-\frac{n}{n+1} & \text { if } i=j \\
\frac{1}{n+1} & \text { if } i>j\end{cases} \\
M=\frac{1}{n+1}\left[\begin{array}{ccccc}
-n & 1 & \ldots & \ldots & 1 \\
1 & \ddots & \ddots & & \vdots \\
\vdots & \ddots & \ddots & \ddots & \vdots \\
\vdots & & \ddots & \ddots & 1 \\
1 & \ldots & \ldots & 1 & -n
\end{array}\right]=\frac{1}{n+1} J_{n-1}-\boldsymbol{I}_{n-1}
\end{gathered}
$$

where $\boldsymbol{I}$ is the identity matrix and $\boldsymbol{J}$ is the matrix full of ones.

As a result, the eigenvalues of $\mathrm{M}$ are:

- -1 , with a multiplicity of $n-2$,

- $\left(\frac{n-1}{n+1}-1\right)=\frac{-2}{n+1}$ with a multiplicity of 1 .

All the eigenvalues of $M$ are strictly negative so $M$ is negative definite. Thus, $\forall x \in \mathbb{R}^{*}, x^{T} M x<0$ and so $\dot{V}(\varepsilon)<0$ for $\varepsilon \neq 0$. Consequently, the longitudinal errors between the robots in the formation converge to zero and the formation is asymptotically stable.

\subsection{Asymmetrical constant coefficients}

Let us consider another particular case where the weights are equal and constant for all robots and define asymmetrical interactions, namely $\left\{\begin{array}{l}\mu_{i-1}^{i}=2 / 3 \\ \mu_{i+1}^{i}=1 / 3\end{array}\right.$ (or vice versa). With this definition, the weight of the preceding elementary control is twice the weight of the following elementary control in the final velocity control law. Another way of seeing this asymmetry is, focusing on a pair $(i-1, i)$ of vehicles, to say that the influence of $i$ on $i-1$ will be half the influence of $i-1$ on $i$.

A stability proving method similar to the one in the previous case is carried out.

Firstly, the determinant of $A$ can be proven to be

$$
\operatorname{det}(A)=\frac{2^{n+1}-1}{3^{n}}
$$

$A$ can be thus inversed and the elements of $A^{-1}$ are

$$
\left\{\begin{array}{l}
a_{i j}^{-1}=\frac{3 \cdot\left(2^{i}-1\right)\left(2^{n-j+1}-1\right)}{2^{n+1}-1} \\
a_{i j j}^{-1}=\frac{3 \cdot 2^{i-j} \cdot\left(2^{j}-1\right)\left(2^{n-i+1}-1\right)}{2^{n+1}-1}
\end{array}\right.
$$

The matrices $M$ and $N$ in the derivative of the Lyapunov function (26) can be explicited

$$
N=D \cdot A^{-1} \cdot C
$$


$A^{-1} . C$ is a vector of dimension $n$ and the $i$ th element is

$$
\begin{aligned}
\left(A^{-1} \cdot C\right)_{i} & =\mu_{0}^{1} \cdot a_{i 1}^{-1}+\mu_{n+1}^{n} \cdot a_{i n}^{-1} \\
& =\frac{2}{3} \frac{3 \cdot 2^{i-1} \cdot\left(2^{1}-1\right)\left(2^{n-i+1}-1\right)}{2^{n+1}-1}+\frac{1}{3} \frac{3 \cdot\left(2^{i}-1\right)\left(2^{n-n+1}-1\right)}{2^{n+1}-1} \\
& =1
\end{aligned}
$$

$A^{-1}$.C is the unity vector and therefore, given the structure of the matrix $D$, it can be easily deduced that

$$
N=D \cdot A^{-1} \cdot C=\mathbf{0}_{(n-1) \times 1}
$$

As for the matrix $M=D \cdot A^{-1} \cdot B$, its dimension is $(n-1, n-1)$, and, for $(i, j) \in\{1,2, . ., n-1\}^{2}$, it is expressed as

$$
\begin{aligned}
& m_{i j}=-\frac{1}{3} a_{i j}^{-1}+\frac{2}{3} a_{i(j+1)}^{-1}+\frac{1}{3} a_{(i+1) j}^{-1}-\frac{2}{3} a_{(i+1)(j+1)}^{-1}= \begin{cases}\frac{2^{i}}{2^{n+1}-1}-1 & \text { if } i=j \\
\frac{2^{i}}{2^{n+1}-1} & \text { otherwise }\end{cases} \\
& M=\frac{1}{2^{n+1}-1}\left[\begin{array}{cccc}
2^{1} & \ldots & \ldots & 2^{1} \\
2^{2} & \ldots & \ldots & 2^{2} \\
\vdots & \ldots & \ldots & \vdots \\
2^{n-1} & \ldots & \ldots & 2^{n-1}
\end{array}\right]-\boldsymbol{I}_{n-1}
\end{aligned}
$$

As a result, the eigenvalues of $\mathrm{M}$ are

- -1 , with a multiplicity of $n-2$,

- $\frac{\sum_{i=1}^{n-1} 2^{i}}{2^{n+1}-1}-1=-\frac{2^{n}+1}{2^{n+1}-1}$ with a multiplicity of 1 .

All the eigenvalues of $M$ are strictly negative so $M$ is Hurwitz. Thus, $\forall x \in \mathbb{R}^{*}, x^{T} M x<0$ and $\dot{V}(\varepsilon)<0$ for $\varepsilon \neq 0$. Consequently, the longitudinal errors between the robots in the formation converge to zero and the formation is asymptotically stable.

This demonstration of stability is here detailed for two specific expressions of relative weights between the two reference robots. However, the same demonstration holds for any expression of constant coefficients. It can be verified by a numerical analysis ${ }^{1}$ that for a given value of the coefficients $\mu$, the matrix $N$ is still null and the matrix $M$ is Hurwitz, thus proving the asymptotic stability of the formation.

\section{Simulation results}

Simulations were conducted to exhibit the applicability of the control laws for a formation of 5 vehicles. In the simulations, each robot is fitted with odometry sensors, an IMU, a RTK-GPS providing a 2-cm accuracy and a WiFi module to communicate with its neighbors, similarly to the sensors equipping the real robotic platforms of the laboratory. The simulator, named Cobaye, emulates data provided to the sensors of the 


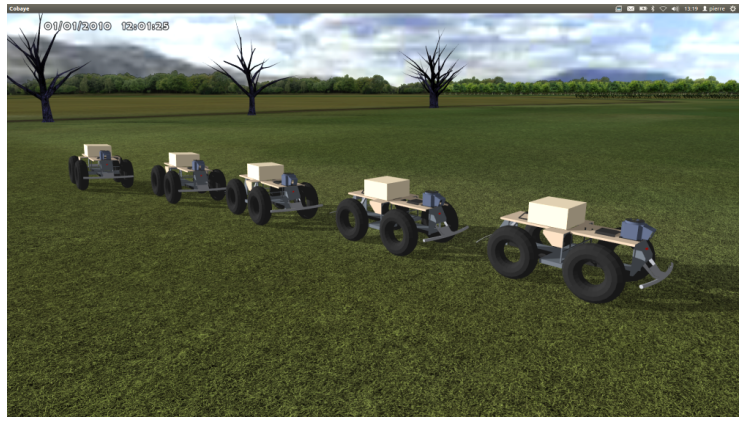

(a) Simulation environment

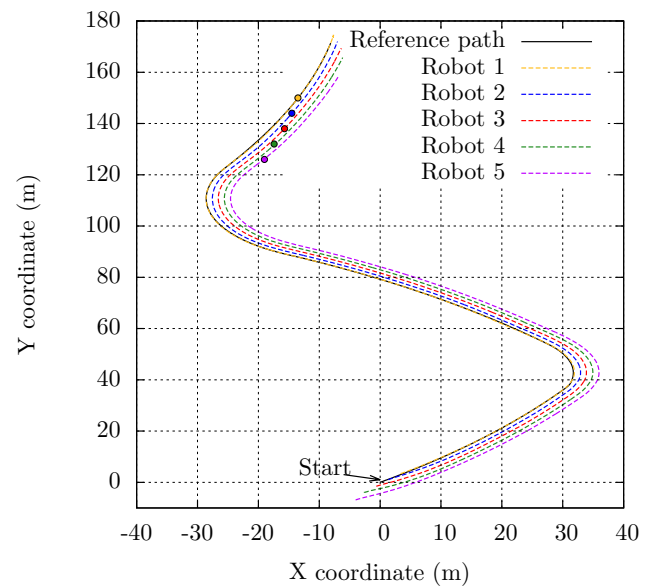

(b) Trajectory of the robots

Figure 4: Illustration of the simulation environment

robots with their accuracy and noises. As for the behavior of the robots, the simulator uses a complete dynamical model and a wheel-ground interaction model to generate the evolution of the robots.

A set of GPS positions has been recorded and represents a path on a flat ground in shape of S with a first bend to the left and a second bend to the right, as illustrated in Figure 4(b). The five robots are initially positioned at the beginning of the reference path, as illustrated in Figure 4(a). The desired formation is wing-shaped with constant lateral and longitudinal distances between robots, as detailed in Table 1. The global desired velocity, known by the first and the last robot, is constant at $3 \mathrm{~m} / \mathrm{s}$.

\begin{tabular}{|l|c|c|c|c|c|}
\hline & Robot 1 & Robot 2 & Robot 3 & Robot 4 & Robot 5 \\
\hline$y_{i}^{\text {des }}(m)$ & 0 & 1 & 2 & 3 & 4 \\
\hline$d_{i / i-1}^{\text {des }}(m)$ & & 6 & 6 & 6 & 6 \\
\hline
\end{tabular}

Table 1: Desired distances in the formation

The lateral servoing of the robots to a desired offset to the trajectory has been previously studied by the authors (see (Lenain et al., 2014)) and is shown to be decoupled from the longitudinal performances. The lateral deviations obtained during the formation control algorithm are compared in Figure 4 and show performances of a few centimeters accuracy. After the initial convergence in $20 \mathrm{~m}$ (defined by the choice of the gains in the control law (7)), these maximal lateral errors occur when the reference trajectory presents sharp curvature variations (at the begining or ending of the curves, curvilinear abscissæ $50 \mathrm{~m}, 75 \mathrm{~m}, 125 \mathrm{~m}$ and $175 \mathrm{~m}$ ). These limited deviations are due to the fact that the desired trajectory has been theoretically generated, and presents discontinuities in the curvature (required to compute control law on steering angle). Such discontinuities lead to the fact that the desired path is not admissible (robots are not able to apply instantaneously a steering angle), and a lateral overshoots appears. Nevertheless, the fleet control stays stable, the lateral control law permits to recover from the discontinuities and the configuration quickly converges back to the desired shape.

Since the lateral performances have already been studied in previous works, let us detail the longitudinal performances, with respect to different selections of coefficients $\mu_{i-1}^{i}$ and $\mu_{i+1}^{i}$. As pointed out in equation (12), these coefficients link each robot $i$ to the previous one $i-1$ and the following one $i+1$, in the longitudinal control. In order to investigate the influence of these coefficients, the path has been tracked three times with

\footnotetext{
${ }^{1}$ A Matlab calculation function for any constant coefficient value may be downloaded on the ftp site: XXXX
} 


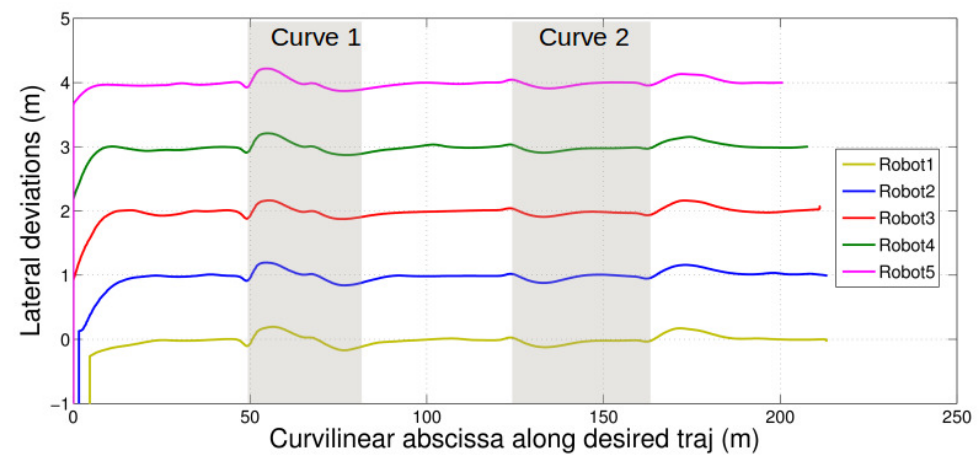

Figure 5: Lateral deviations of each robot

different combination coefficients in the control laws $\left(\mu_{i-1}^{i}=1, \mu_{i-1}^{i}=2 / 3\right.$ and $\left.\mu_{i-1}^{i}=1 / 2\right)$, and the results are presented in figures $6(\mathrm{a})$ to $6(\mathrm{c})$. In order to illustrate the stability of the whole fleet, the results present the cumulated longitudinal error of each robot with respect to the first one in Figure 6. At the entry of the first curve (to the left, at curvilinear abscissa $45 \mathrm{~m}$ ), the robots 2 to 5 are on the outside of the curve and must accelerate to keep the interdistance. Similarly, at the abscissa $120 \mathrm{~m}$, the robots enter the second curve, to the right, and must slow down.

With the referencing only to the preceding robot (with the coefficients $\mu_{i-1}^{i}=1, \mu_{i+1}^{i}=0$ the second referencing is not considered, see Figure 6(a)), a transient error appears when the curvature changes, since the velocity of the robots must change sharply. The errors are corrected when the trajectory curvature comes back constant. The longitudinal regulation of a vehicle with respect to the previous one is precise within $\pm 0.15 \mathrm{~m}$ but on the overall, as these errors accumulate, the total regulation error between the first and the last robot of the fleet is of $0.5 \mathrm{~m}$ in the curves (and even $0.9 \mathrm{~m}$ at the entry of the first curve).

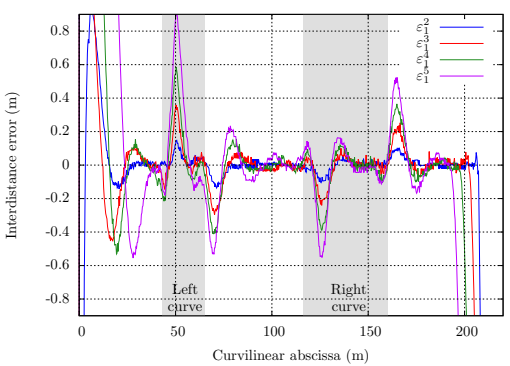

(a) Reference to previous robot only

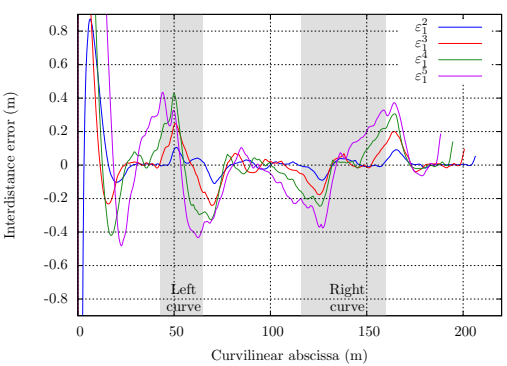

(b) $\mu_{i-1}^{i}=1 / 3, \mu_{i+1}^{i}=2 / 3$

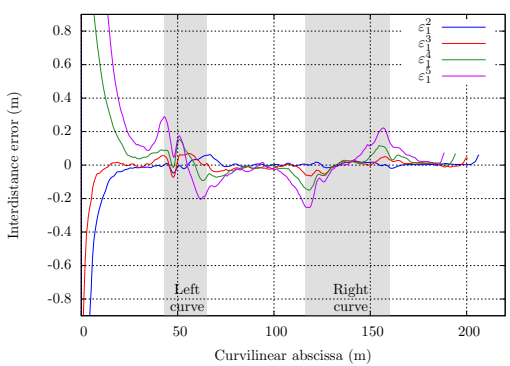

(c) $\mu_{i-1}^{i}=\mu_{i+1}^{i}=1 / 2$

Figure 6: Comparison of longitudinal errors for different choices of combination coefficients

In the second simulation, which results are presented in Figure 6(b), the following robot is now accounted for, but with a relative weight in the control law still half as much as the preceding robot's weight $\left(\mu_{i-1}^{i}=1 / 3, \mu_{i+1}^{i}=2 / 3\right)$. The addition of the second referencing permits to decrease the amplitude of the errors to less than $0.4 \mathrm{~m}$ for the whole fleet but it also slows down the variations, which increases the standard deviation of the error (the formation stays less to its desired configuration).

In the last simulation, the relative weight of the following robot in the velocity control is increased to be equal to the weight of the preceding robot so that each robot is equally "pulled" by the preceding robot as much as "pushed" by the following one. In this case, the velocity changes (due to the curvature evolutions) are passed on consistently by both elementary control laws, which permits to widely reduce the accumulation of errors in one direction. As a result, the longitudinal errors for the whole fleet are reduced to $\pm 0.25 \mathrm{~m}$ (see 
Figure $6(\mathrm{c})$ ), a precision twice better than the one obtained with a referencing to the previous vehicle only.

\section{$5 \quad$ Experimental results}

The simulations conducted have permitted to show the contribution of the proposed approach to stabilize a formation of numerous vehicles travelling along a path, accounting for the conditions of evolution. To evaluate the performances of the algorithms for agricultural needs, differents full-scale experiments have also been realized. Due to technical reasons, the experiments have been conducted with three vehicles of different kinds and with different conditions of evolution to validate the generic nature of the approach.

\subsection{Results with heterogeneous robots}

In the first experiment presented, the fleet regulation approach is applied to a formation of three autonomous vehicles, focusing on the robustness to the heterogeneity of the vehicles. The first autonomous vehicle is a commercial 12-tons tractor (fully automated) and the two next robots are lighter experimental electricallydriven platforms, as illustrated in Figure 7. Their characteristics are detailed in Table 2. All of them are fitted with RTK-GPS receiver, providing a position accurate within $2 \mathrm{~cm}$ at a $10 \mathrm{~Hz}$ frequency, an IMU and a wireless communication module (based on the standard IEEE 802.11p). Also, within the control algorithms, data are extrapolated to account for the communication delays observed in real experiments. It is possible since the dates of localization are transmitted along with the data with the communication module.

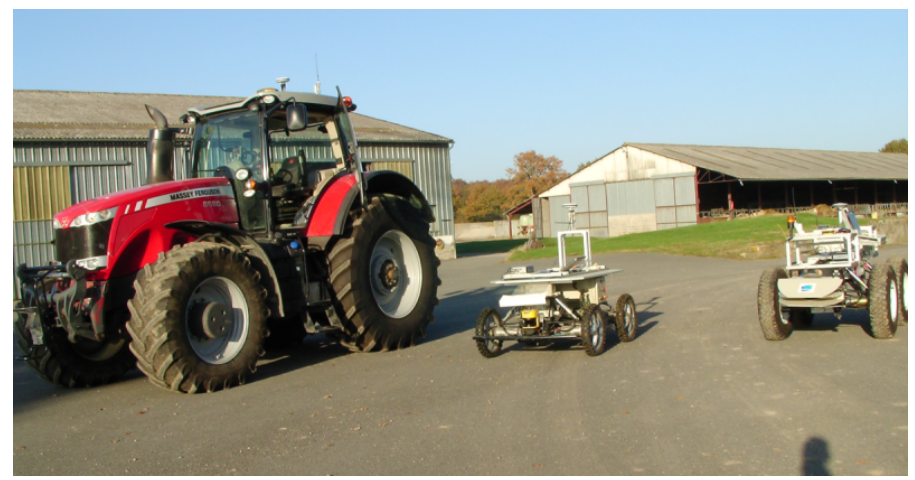

Figure 7: Illustration of the experimental vehicles

\begin{tabular}{|c||c|c|c|}
\hline & Tractor & 1st follower & 2 nd follower \\
\hline \hline Weight & $10300 \mathrm{~kg}$ & $600 \mathrm{~kg}$ & $420 \mathrm{~kg}$ \\
\hline Trackwidth & $3,1 \mathrm{~m}$ & $1,2 \mathrm{~m}$ & $1,2 \mathrm{~m}$ \\
\hline Maximal steering angle & 48 & 20 & 25 \\
\hline Steering angle settling time & $1,6 \mathrm{~s}$ & $0,4 \mathrm{~s}$ & $0,6 \mathrm{~s}$ \\
\hline Maximal speed & $14 \mathrm{~m} \cdot \mathrm{s}^{-1}\left(50 \mathrm{~km} \cdot \mathrm{h}^{-1}\right)$ & $8 \mathrm{~m} \cdot \mathrm{s}^{-1}$ & $4 \mathrm{~m} \cdot \mathrm{s}^{-1}$ \\
\hline
\end{tabular}

Table 2: Main characteristics of the experimental vehicles

The path to track, shown in Figure 8(a), had been recorded beforehand and is loaded by the 3 robots at the initialization of the algorithm. The robots travel on a tarred path and the desired velocity travel is of $3 \mathrm{~m} / \mathrm{s}$. The desired formation is line-shaped, except for the first robot, for which a lateral offset of $0.6 \mathrm{~m}$ is set from the beginning of the trajectory up to an achieved distance of $155 \mathrm{~m}$ (at the end of last curve). For the desired longitudinal behavior, a constant distance of $30 \mathrm{~m}$ is set all along the experiment ${ }^{2}$.

\footnotetext{
${ }^{2} \mathrm{~A}$ video of this experiment and similar tests may be downloaded on the ftp site: XXXX
} 
This experimental configuration with a line formation and a trajectory presenting curves and straight line parts will be classicaly used in the agricultural applications promoted for transportation (commutation between farm and field) or for contouring in the field with different successive works realized by each autonomous vehicle.

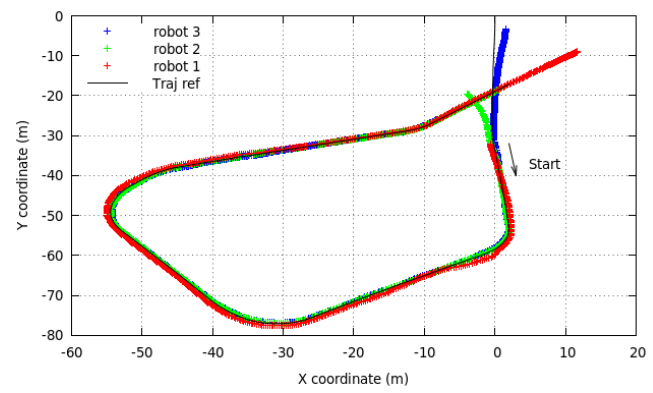

(a) Trajectories in absolute frame

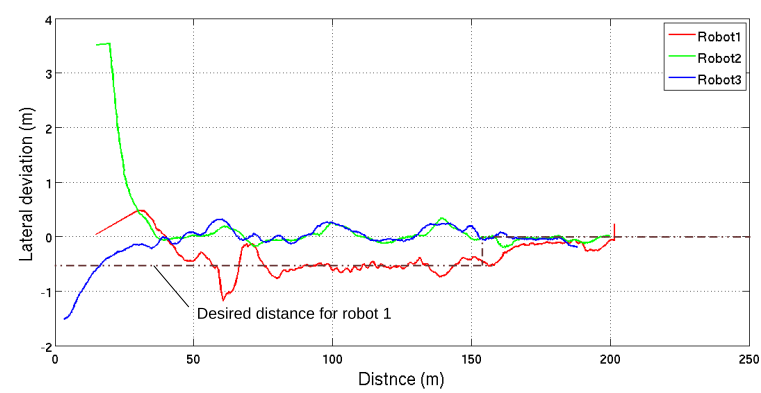

(b) Lateral deviation

Figure 8: Trajectory of the robots and lateral deviation

From a lateral servoing point of view, it can be noticed that after an initialization distance of $20 \mathrm{~m}$, each robot is able to reach its desired lateral deviation and to maintain it within an accuracy of $\pm 15 \mathrm{~cm}$, which is consistent with agricultural task requirements. The first robot (a commercial farm tractor) admits some overshoots at the end of initial convergence (which corresponds to the first right turn), due to the settling time of its steering actuator. Indeed, it is equipped with an hydraulic valve which settling time is bigger than the electric actuators of the two other robots. For homogeneity reasons, the same tuning gains in control law (7) have been chosen for all robots, which is optimal for electrical robots but for the automated farm tractor, the real response dynamic leads to some small oscillations when having important error to compensate in lateral deviation. This first robot is nevertheless able to follow the desired lateral distance, as it converges to $-0.6 \mathrm{~m}$ up to $155 \mathrm{~m}$ and reach 0 , when the desired lateral distance to the trajectory is set to zero, as depicted in Figure 8(b).

Regarding the longitudinal behavior, each robot proportionally combines the velocity control referenced to the previous vehicle and the control referenced to the following vehicle, i.e. $\mu_{i-1}^{i}=\mu_{i+1}^{i}=\frac{1}{2}$ in the combination law (12).

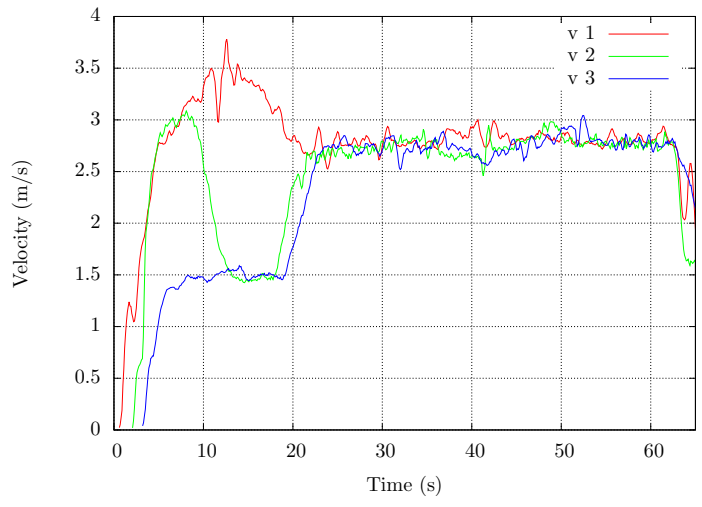

(a) Velocities with proportional referencing

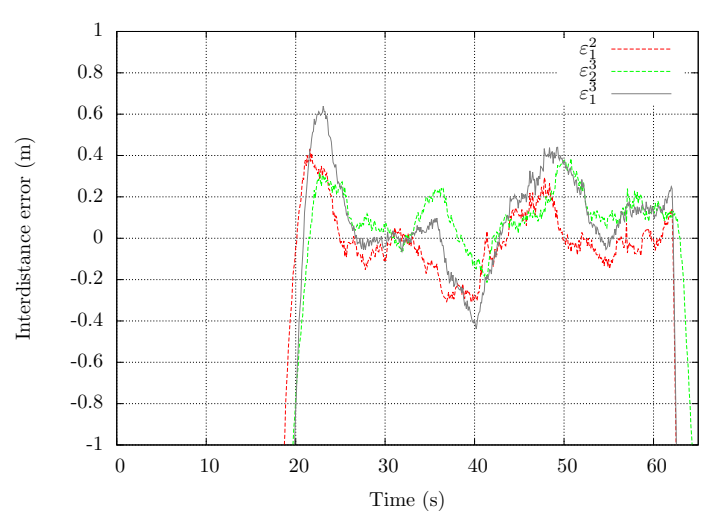

(b) Interdistance error with proportional referencing

Figure 9: Longitudinal results of the 3-vehicle path tracking

The results of the path tracking are presented in Figure 9. At the beginning, the vehicles are positioned longitudinally spaced of around $10 \mathrm{~m}$. They are too close to each other so the first vehicle is "pushed" by 
its following neighbor to a velocity higher than the desired travel velocity $v^{C}=3 \mathrm{~m} / \mathrm{s}$ in order to expand the gap between the robots. Similarly, at the beginning the last vehicle is too close to the middle one and combines the desired travel velocity to the null control velocity (waiting for the previous robot to move forward and reach the desired interdistance); as a result, the last robot initial velocity is of $1.5 \mathrm{~m} / \mathrm{s}$. Finally, the robots converge to the desired interdistance of $30 \mathrm{~m}$ in $20 \mathrm{~s}$ and their velocity converges to the desired travelling speed. The convergence distance is set by the acceleration capabilities of the vehicles. Here the tractor is the limiting vehicle as, with its inertia, it is the slowest to start and accelerate up to $3.5 \mathrm{~m} / \mathrm{s}$. The following robots, the lighter electrically-driven platforms which are more reactive, limit their accelerations to be consistent with the tractor dynamic. Then, during the path tracking the longitudinal regulation permits to keep the interdistance with a maximal regulation error of $0.3 \mathrm{~m}$, and a maximal interdistance error between the head and the tail of the formation of $0.4 \mathrm{~m}$. Moreover, the stability of the longitudinal regulation permits to keep the constant desired velocity for all robots and avoid the stop-and-go effects due to error accumulation.

\subsection{Results in agricultural work configuration}

The proposed control architecture for formation control of several mobile robots appears to be generic and may be applied easily to different kind of vehicles, since the only parameter to be known is the vehicle wheelbase $L$. The possible applications may then fit several agriculture tasks, in different configurations of use. As an example, this section proposes experiments with three agricultural tractors. The first one is manually controlled and generates on-line a reference trajectory as a set of GPS positions. The two following tractors are autonomous; their commands are calculated using the proposed algorithms and sent to the actuators on the CAN bus of the tractor. The same equipment as the one used for previous experimentation is used. A RTK GPS supplies position and heading, while wireless communication modules allow the exchange of data between tractors. As can be seen in Figure 10 extracted from the joint video ${ }^{3}$, the second vehicle is equipped with an implement acting on the soil during straight line parts.

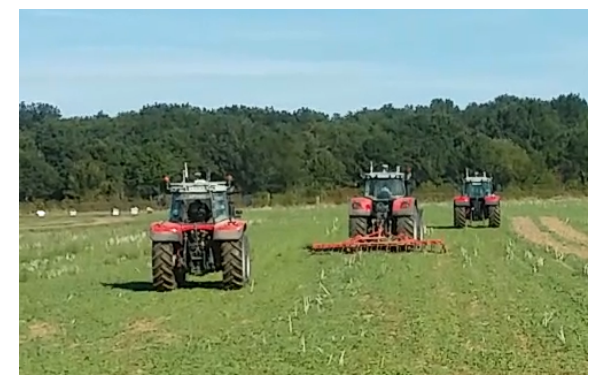

Figure 10: Experimentation in autonomous agricultural work configuration

The proposed control architecture is embedded on the two following tractors. The human driver of the first vehicle is able to tune the desired lateral distance to the path of the two followers. In this experiment, the leader is driven in a tubble ploughed field and realizes a trajectory representative of an agricultural work (see black curve in Figure 11(a)). It is composed of straight lines with half turns at the end of the rows. The length of the leader trajectory is equal to $800 \mathrm{~m}$ and has been achieved at an average velocity of $1.6 \mathrm{~m} / \mathrm{s}$ (almost $6 \mathrm{~km} / \mathrm{h}$ ), with a stop point at the end of the second half turn. This lead the two followers to stop during curve, which is a challenging situation for the algorithms. The stop points of the vehicles are marked in Subfigure 11(b). In order to limit the area of experiments, two rows are used several times. As a result the complete trajectory of the three robots is split into two subfigures (see Figure 11), each defining half of the complete trial (representing a traveled distance by the leader of $400 \mathrm{~m}$ ).

During the straight line parts, the driver tunes a desired lateral distance for the followers, so that each tractor is positionned at a distance of one width (of the tractor or the implement) from the previous tractor. This

\footnotetext{
${ }^{3}$ available on the ftp website: XXX
} 


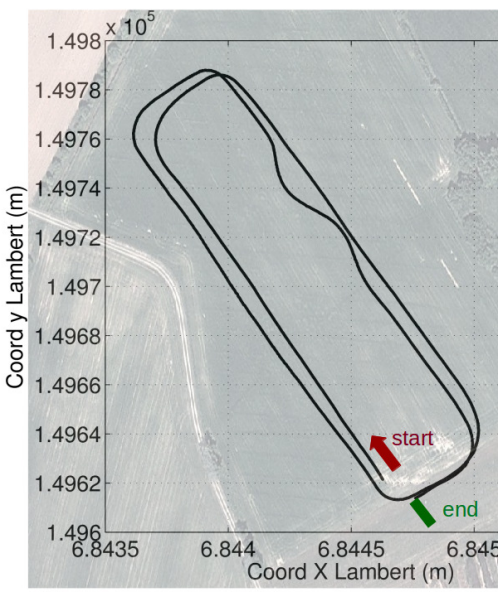

(a) Whole trajectory of the leader

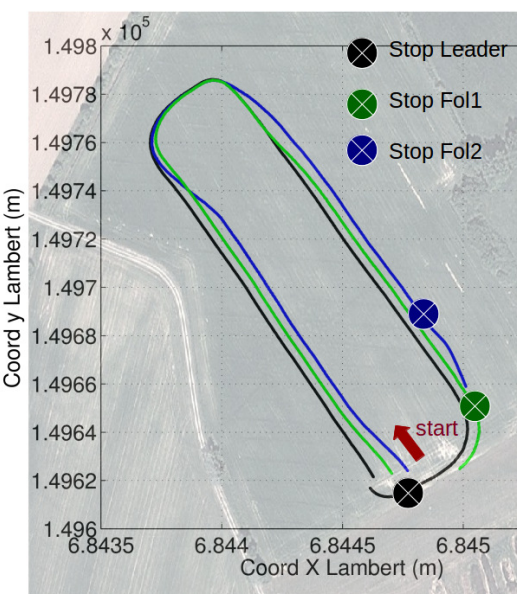

(b) First part (Leader and followers)

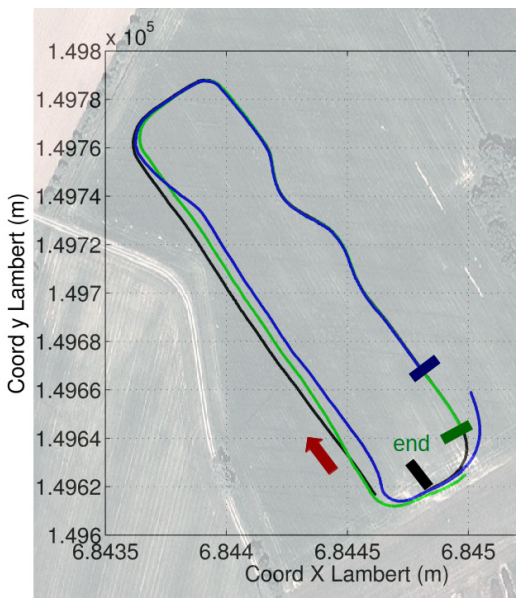

(c) Second part (Leader and followers)

Figure 11: Trajectory of the robots

corresponds to a classical "wing" configuration in agriculture where the tractors achieve parallel work and cooperate to cover the whole field in a minimum of time. During half turn, the lateral distance is set to zero in order to limit dead zone in field borders.In the last line, the manually driven leader diverts its trajectory and achieves some sinusoidal deviation, similarly to the reaction of a driver realizing an obstacle avoidance. During this last part, the desired lateral distances of the follower are set to zero. From a longitudinal point of view, a constant interdistance of $30 \mathrm{~m}$ is set all along the test.

Beyond the results pointed out on the trajectory, let us consider the deviation obtained on lateral and longitudinal servoing, depicted in Figure 12. Lateral distances are reported in Figure 12(a) together with the desired set points which are changing during the experiment. It can be noticed that the actual lateral deviation of the first (in green plain line) and the second follower (in blue dashed line) indeed reach their respective set point after the theoretical distance of convergence of $25 \mathrm{~m}$ set by the tuning of the control gains.

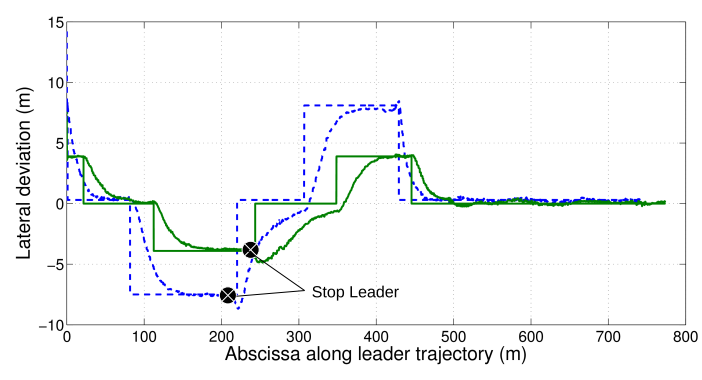

(a) Lateral deviation of followers

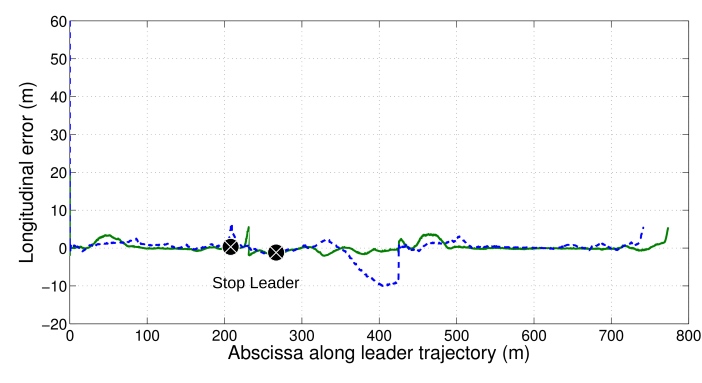

(b) Longitudinal positioning error of the followers

Figure 12: Deviations obtained during second experiment

After the settling distance, the lateral error is well regulated, within an accuracy of few centimeters, and the variation of the trajectory shape in the sinusoidal part (after curvilinear abscissa $550 \mathrm{~m}$ ) do not affect the accuracy of the regulation, which performances are described in Table 3. The most challenging situation encountered for the lateral regulation is the stopping part. Indeed, as the halt of the leader occurs in the second half turn, it coincides with the transition from the wing-shaped formation (non null lateral deviation) to the line formation for the headland maneuver. As a result, the follower tractors steer while braking which 


\begin{tabular}{|r|r|c|c|}
\hline & & Follower 1 & Follower 2 \\
\hline \hline Lateral Error & Mean & $0.02 \mathrm{~m}$ & $0.14 \mathrm{~m}$ \\
\hline & Standard deviation & $-0.08 \mathrm{~m}$ & $0.19 \mathrm{~m}$ \\
\hline Longitudinal Error & Mean & $0.12 \mathrm{~m}$ & $-0.40 \mathrm{~m}$ \\
\hline & Standard deviation & $0.57 \mathrm{~m}$ & $0.75 \mathrm{~m}$ \\
\hline
\end{tabular}

Table 3: Statistic on longitudinal and lateral error during second experiment

creates transient dynamic effects unaccounted for in the control laws.

On the longitudinal side, after the first convergence phase, and excluding the transient phase of stopping and restarting of the formation, the velocity control law permits to keep the autonomous following tractors at their desired position (30 $\mathrm{m}$ to the previous tractor) during the nominal part of the evolution with an accuracy of a few decimeters, as is depicted in Figure 12(b) and pointed out in Table 3. Despite the uneven surface of evolution, the curves and the modifications of lateral positionning, the followers adapt their velocity consistently to maintain their interdistance to their desired value.

About the transient phase of halt of the formation, it can be noticed that if the first follower handles it prefectly (there is no deterioration of the longitudinal regulation accuracy in this zone), the second follower's performances are deteriorated (a drift after curvilinear abscissa $325 \mathrm{~m}$ is observed reaching $-10 \mathrm{~m}$ at curvilinear abscissa $420 \mathrm{~m}$ ). This difference comes mainly from material considerations, namely the acceleration capabilities of the tractors. Indeed, if the first two tractors are similar, the second follower is an older tractor on which the gearbox cannot be controlled. As a result, the second follower's acceleration is slower than the leader's and the first follower's and the former cannot follow the latters. Moreover after the tractors restart, in the acceleration phase they exit the headland maneuver area and the formation shape is turned back to a wing, the lateral setpoint of the second follower is increased to $8 \mathrm{~m}$ at the curvilinear abscissa $310 \mathrm{~m}$ as it can be seen in Figure 12(a). As a result, to reach this new desired lateral position, the second follower has to accelerate even more, which exceedes widely the aceleration capability of the real tractor.

The experiment presented is representative of the behaviors observed during several experimentation campaigns in different evolution conditions as it permits to point out both the strength of the proposed approach for a stable path tracking in formation with varying desired formation shape in agricultural context, for the lateral as well as for the longitudinal regulation, and to exhibit the limitations of the current implementation. On the bright side, the addition of an implement behind the second tractor is well accounted for as its effect on the lateral evolution of the tractor is interpreted as skidding and compensated in the steering control law. About the velocity regulation, the implement used, a harrow, produces a force light enough so that the power of the tractor can maintain the desired velocity and keep an accurate interdistance. In case of use of a deeper working implement, such as a plow, its effect on the longitudinal evolution may become non negligible and an accurate regulation may benefit from the estimation of the longitudinal sliding.

On the other side, limitations between the theoretical regulation and the experimental evolution have been exhibited. Firstly, sharp velocity variations during steering parts have led to transient lateral error because of dynamical effects ignored in the control laws (based on a theoretical decoupling between lateral and longitudinal regulation). But mainly, the principal limitation highlighted comes from the limited acceleration of the real tractors Because of the settling time in the combustion engine of the tractors, the longitudinal regulation is less accurate than lateral regulation (hydraulic cylinder). The performances are thus depreciated compared those one obtained with electrical robots. Moreover, this experiment is a challenging test case since speed variation and on-line modification of the lateral set point have been achieved. A better low-level control loop for throttle will help reducing the longitudinal error. Nevertheless, the lateral accuracy with heavy automated farm tractor is of few centimetres, while longitudinal errors does not exceed few decimetres (less than half of vehicle wheelbase). This level of error is satisfactory in order to control farm tractors in a dedicated formation for cooperative field work. 


\section{Conclusion}

In this paper, we study the path tracking in formation of a fleet of mobile robots. The model of the vehicles includes the effects of the evolution in a natural environnement by means of sideslip angles. Using this extended model, the steering control law permits to regulate precisely the lateral deviation to the trajectory of each robot to a desired offset, even if this one is changed during the experiment. The velocity control strategy in the fleet defined consists, for each robot, in a linear combination of the two velocity commands regulating the interdistance to the two closest neighbors. A Lyapunov approach applied to different cases, a 3 -vehicle formation and a $n$-vehicle formation with constant symmetrical and asymmetrical interaction coefficients, has proven the stability of the formation. This control has been tested through simulation with a 5-vehicle formation and through experiments using 3-vehicle formations. The application in off-road context of the control strategy developped have confirmed the stability of the formation path tracking, highlighting the reduction of the error accumulation by a factor of two compared to a classical leader-follower strategy on a larger fleet. This strategy has been successfully applied to heterogeneous formations of electrical robots and autonomous huge farm tractor propelled with combustion engine. In a constant or slow varying formation, a high level of accuracy may be expected, whatever the (variable) kind of ground and the trajectory shape are, demonstrating the stability and robustness of the approach.

Future work will focus, on the theoretical side, on the expansion of the stability proof of the bidirectional control law with variable coefficients in order to characterize and on-line adapt the behavior of the formation. From the applicative point of view, a more precise model of the low-level throttle behavior will be developped and added in the control loop to account for the acceleration capabilities of the real tractors and so enhance the accuracy of the longitudinal fleet servoing. The use of local perception (such as laser rangefinder) will also be studied in order to reduce the required equipment related to sensors cost.

\section{Acknowledgment}

This work has received the support of French National Research Agency under the grant number ANR-14CE27-0004 attributed to Adap2E project (adap2e.irstea.fr). It has also been sponsored through the RobotEx Equipment of Excellence (ANR-10-EQPX-44), by the European Union through the program Regional competitiveness and employment 2007-2013 (ERDF Auvergne Region), and by the Auvergne Region

\section{References}

Antonelli, G., Arrichiello, F., Caccavale, F., and Marino, A. (2013). Decentralized centroid and formation control for multi-robot systems. In IEEE International Conference on Robotics and Automation (ICRA), pages $3511-3516$.

Balch, T. and Arkin, R. C. (1998). Behavior-based formation control for multirobot teams. IEEE Transactions on Robotics and Automation, 14(6):926-939.

Baras, J., Tan, X., and Hovareshti, P. (2003). Decentralized control of autonomous vehicles. 42nd IEEE International Conference on Decision and Control, 2:1532-1537.

Blackmore, C. (2014). Learning to change farming and water management practices in response to the challenges of climate change and sustainability. Outlook on AGRICULTURE, 43(3):173-178.

Campion, G., Bastin, G., and d'Andréa Novel, B. (1996). Structural properties and classification of kinematic and dynamic models of wheeled mobile robots. IEEE Transactions on Robotics and Automation, $12(1): 47-62$.

El-Mikkawy, M. and Karawia, A. (2006). Inversion of general tridiagonal matrices. Applied Mathematics Letters, 19(8):712-720. 
Farrokhsiar, M. and Najjaran, H. (2012). An unscented model predictive control approach to the formation control of nonholonomic mobile robots. In IEEE International Conference on Robotics and Automation (ICRA), pages $1576-1582$.

Guillet, A., Lenain, R., and Thuilot, B. (2013). Off-road path tracking of a fleet of WMR with adaptive and predictive control. In IEEE/RSJ International Conference on Intelligent Robots and Systems (IROS), pages $2855-2861$.

Guillet, A., Lenain, R., Thuilot, B., and Martinet, P. (2014). Adaptable robot formation control: adaptive and predictive formation control of autonomous vehicles. IEEE Robotics 83 Automation Magazine, 21(1):28-39.

Khatir, M. and Davison, E. (2004). Decentralized control of a large platoon of vehicles using non-identical controllers. In Proceedings of the American Control Conference, volume 3, pages 2769-2776.

Klančar, G., Matko, D., and Blažič, S. (2011). A control strategy for platoons of differential drive wheeled mobile robot. Robotics and Autonomous Systems, 59(2):57 - 64 .

Kowdiki, K. H., Barai, R. K., and Bhattacharya, S. (2012). Leader-follower formation control using artificial potential functions: A kinematic approach. In IEEE International Conference on Advances in Engineering, Science and Management (ICAESM), pages 500-505.

Lafferriere, G., Williams, A., Caughman, J., and Veerman, J. (2005). Decentralized control of vehicle formations. Systems \&6 Control Letters, 54(9):899-910.

Lenain, R., Thuilot, B., Cariou, C., and Martinet, P. (2006). High accuracy path tracking for vehicles in presence of sliding: Application to farm vehicle automatic guidance for agricultural tasks. Autonomous Robots, 21(1):79-97.

Lenain, R., Thuilot, B., Guillet, A., and Benet, B. (2014). Accurate target tracking control for a mobile robot: a robust adaptive approach for off-road motion. In IEEE International Conference on Robotics and Automation (ICRA), pages 2652-2657.

Qian, D., Tong, S., Guo, J., and Lee, S. (2015). Leader-follower-based formation control of nonholonomic mobile robots with mismatched uncertainties via integral sliding mode. Proceedings of the Institution of Mechanical Engineers, Part I: Journal of Systems and Control Engineering, 229:559-569.

Ren, W. and Beard, R. (2004). Decentralized scheme for spacecraft formation flying via the virtual structure approach. In Journal of Guidance, Control, and Dynamics, volume 27, pages 73-82.

Samson, C. (1995). Control of chained systems application to path following and time-varying pointstabilization of mobile robots. IEEE Transactions on Automatic Control, 40(1):64-77.

Wang, D. and Low, C. (2007). An analysis of wheeled mobile robots in the presence of skidding and slipping: Control design perspective. In IEEE International Conference on Robotics and Automation (ICRA), pages 2379-2384, Roma, Italy.

Yamaguchi, H., Arai, T., and Beni, G. (2001). A distributed control scheme for multiple robotic vehicles to make group formations. Robotics and Autonomous Systems, 36(4):125 - 147.

Zhang, Y., Kosmatopoulos, E. B., Loannou, P. A., and Chien, C. C. (1999). Autonomous intelligent cruise control using front and back information for tight vehicle following maneuvers. IEEE Transactions on Vehicular Technology, 48(1):301-318. 